\title{
Philosophiques
}

\section{Les idées politiques de d'Holbach et la Déclaration des Droits de 1789}

\section{Josiane Boulad-Ayoub}

Volume 18, numéro 2, automne 1991

URI : https://id.erudit.org/iderudit/027155ar

DOI : https://doi.org/10.7202/027155ar

Aller au sommaire du numéro

Éditeur(s)

Société de philosophie du Québec

ISSN

0316-2923 (imprimé)

1492-1391 (numérique)

Découvrir la revue

\section{Citer cet article}

Boulad-Ayoub, J. (1991). Les idées politiques de d'Holbach et la Déclaration des Droits de 1789. Philosophiques, 18(2), 123-137. https://doi.org/10.7202/027155ar
Résumé de l'article

À partir du Système social et de la Politique naturelle du baron d'Holbach, l'auteur dégage les éléments constitutifs du modèle éthico-politique que ces ouvrages dessinent et en déterminent la valeur novatrice par rapport à l'idéologie sociopolitique de l'époque; la seconde partie de l'article examine la Déclaration des Droits de 1789 pour retracer dans quelle mesure la pensée politique de d'Holbach aura influencé la rédaction de ses articles. 


\title{
LES IDÉES POLITIQUES DE D'HOLBACH ET LA DÉCLARATION DES DROITS DE 1789
}

\author{
par Josiane Boulad-Ayoub
}

RÉSUME. À partir du Système social et de la Politique naturelle du baron d'Holbach, l'auteur dégage les éléments constitutifs du modèle éthico-politique que ces ouvrages dessinent et en déterminent la valeur novatrice par rapport à l'idéologie sociopolitique de l'époque; la seconde partie de l'article examine la Déclaration des Droits de 1789 pour retracer dans quelle mesure la pensée politique de d'Holbach aura influencé la rédaction de ses articles.

ABSTRACT. The author brings out d'Holbach's ethical and political model of thought in order to analyze his revolutionary value with respect to the political and societal context. In conclusion the relationships between the ideological thought of $\mathrm{d}^{\prime}$ Holbach and the articles of the Déclaration des Droits will be examined.

Nous voudrions ici, en nous appuyant principalement sur le Système social et sur les IX Discours de la Politique Naturelle ou discours sur les vrais principes $d u$ gouvernement du baron d'Holbach, dégager les éléments constitutifs du modèle éthico-politique que ces ouvrages dessinent et en déterminer la valeur novatrice par rapport à l'idéologie socio-politique de l'époque; la seconde partie de notre exposé examinera la Déclaration des Droits de 1789 pour retracer dans quelle mesure la pensée politique de $\mathrm{d}^{\prime}$ Holbach aura influencé la rédaction de ses articles. On se 
demandera alors, en termes de catégories et d'enjeux idéologiques, quelles sont les marques laissées par le "parti des Philosophes » sur l'organisation du monde en train de se constituer et dont ce discours solennel aux débuts de la Révolution enregistre les principes et les valeurs qui devront la guider. Pièce à verser au dossier des relations discursives entre Philosophes et Révolutionnaires, notre analyse précisera les aspects conceptuels et idéologiques de cette "rencontre" entre le Sujet tel que le situe dans la société française d'Ancien Régime le libéralisme politique de $d^{\prime}$ Holbach et le Citoyen appelé à vivre dans l'État de droit que la toute neuve Assemblée Nationale travaille à établir.

\section{Le modèle politique de $\mathrm{d}^{\prime} \mathrm{Holbach}$}

D'Holbach, développant dans son premier grand livre les principes fondamentaux du matérialisme moderne, entendait, au nom de la Raison et de l'expérience, fournir à l'arsenal théorique des Philosophes une explication "naturelle » du monde et de l'homme; aussi bien en établissant les fondements de leurs revendications politiques contre la «religion» et le "gouvernement», voulait-il aider au triomphe du "parti de l'humanité " et au succès des objectifs de la lutte idéologique: faire effectivement " rentrer le genre humain dans ses droits". Pour des êtres gouvernés par la Nature et soumis à ses lois, les mêmes pour tous, tout devait se ramener à une "morale simple et naturelle, fondée sur leurs besoins", à une "politique appuyée sur des intérêts sensibles ${ }^{1}$. Voici le programme théorique que la lutte des Philosophes répandait bon train pour changer les façons de penser et d'agir. L'homme de la raison future comme l'appelera le Système social, fait pour fuir la peine et rechercher le plaisir, soucieux d'assurer par des lois justes le bonheur de la société, devenait ainsi le premier artisan de son bien-être ou de son mal-être.

Les thèses physiques-ontologiques que d'Holbach a établies dès le Système de la Nature dictent également les options théoriques sous-jacentes à l'élaboration du modèle politico-social. Une même volonté idéologique les anime: en finir avec les superstitions et le fanatisme de la religion comme avec la tyrannie d'un pouvoir absolu. Ce type de religion, cette forme de gouvernement offensent la raison: les rapports individuels et collectifs que supposent ces organisations ne correspondent tout simplement plus ni à la réalité des rapports sociaux ni à la vérité de la nature de l'être humain. 
De même que les forces de la nature, c'est-à-dire "le temps, l'espace, la matière, le mouvement ne sont ni bons ni méchants ", de même on affirmera que les passions, les forces qui déterminent l'être humain comme tel, ne sont ni bonnes ni mauvaises mais nécessaires. Les préceptes du système moral, les règles du système politique seront définies comme des suites nécessaires de l'amour que l'homme a pour lui-même. Ce n'est pas en comptant sur une religion dont les "révélations" varient d'un climat du globe à l'autre, mais sur l'éducation de la raison que l'on habituera les hommes à suivre en tout la règle primordiale $\mathrm{du}$ bonheur et à l'appliquer dans les limites du simple jugement. Force naturelle, le bonheur oblige tout être sensible et raisonnable à prendre les moyens d'où dépend la conservation de son être et à fuir tous les excès dont l'effet serait de rendre son existence douloureuse.

Cette morale dont le but est le bonheur, cet eudémonisme auquel revient la morale naturelle, est, $d^{\prime}$ une part, une morale rationnelle et scientifique puisque les devoirs qu'elle entraîne se fondent sur l'essence de l'homme et sur la connaissance des lois qui le gouvernent; c'est, d'autre part, une morale pragmatique qui réévalue l'action bonne et juste selon le critère de l'utilité: la vertu devenant ce qui s'avère par le jugement utile à la réalisation de son bonheur et de celui de son semblable:

Détrompe-toi donc, Enfant de la nature, de ces rapports fictifs que l'on suppose entre toi et le pouvoir inconnu que l'ignorance a créé, et que l'enthousiasme a revêtu de mille qualités incompatibles. Sois raisonnable, voilà ta religion; sois vertueux, voilà pour toi le chemin de la félicité. Deviens utile aux autres, voilà le moyen de leur plaire, et de les engager à seconder tes projets; ne te nuis point à toi-même, voilà ce que se doit un être raisonnable 3 .

La politique ou plus exactement le système politique sera relié au système moral par le biais de la redéfinition qu'opère $d^{\prime}$ Holbach de la nature d'une organisation sociale. La vie en société trouve son origine dans ce désir de bonheur qui caractérise l'homme. De la même façon que tout homme tend au bonheur, de même cette tendance le poussera à se réunir en société pour être heureux. Le postulat ontologique de l'unité du système naturel et du système social se décompose à l'intérieur du système social comme celui de l'unité des déterminations de l'homme sous son aspect individuel et sous son aspect collectif. Un tel postulat qui, de manière identique, fonde sur le bonheur la morale individuelle et la morale collective, permettra de fixer aussi la mesure de leur efficacité. Et d'Holbach d'écrire: 
"Ainsi la société est un assemblage d'hommes, réunis par leurs besoins, pour travailler de concert à leur conservation et à leur félicité commune ${ }^{4}$ ». Mais en même temps, en ce qui concerne les rapports entre morale et politique, cela transforme la politique en « une morale appliquée au gouvernement des Etats ${ }^{5}$. Avec le bonheur mis au fondement du pacte social ${ }^{6}$, $l^{\prime}$ action sociale signifie, sous le critère de l'utile, une pratique vertueuse conduisant au bonheur de tous dès lors qu'elle s'identifie aux intérêts propres des humains. Morale et politique sont donc liées par un rapport réciproque selon lequel la morale donne aux hommes les moyens spéculatifs pour découvrir cette interrelation entre l'utile (l'intérêt naturel) et le vertueux (l'intérêt culturel) qui assure leur bonheur alors que la politique s'emploie à établir les moyens (lois, coercition, encouragement par les honneurs ou autres bienfaits) de sa pratique effective dans la vie en société car «nul [citoyen] ne peut être heureux sans la vertu?".

Ce que nous devons, par conséquent, attendre de la politique, $c^{\prime}$ est ce que nous devons attendre de la morale mais à une échelle étendue à la collectivité qui forme une nation: gouverner, puisque la politique est l'art de gouverner les hommes réunis en société, c'est appliquer au corps social les préceptes de la morale et faire observer les lois tant naturelles que civiles qui, du point de vue du critère de l'utilité, clé du bonheur social bien compris, s'avèrent nécessaires au maintien de l'ordre existant (conservation du système formé par telle société particulière) comme à la jouissance tranquille des bienfaits qu'apportent la vie en société, la justice, les secours, la protection des fruits du travail, la sûreté. Autrement dit, les forces naturelles qui déterminent un système politique sont "les passions" des hommes et tout l'art du politique consiste, faisant cap sur le bonheur, à maintenir l'équilibre entre les lois de la nature et les lois civiles, lesquelles réglent les "passions " des citoyens d'un même État et les redirigent vers le bien commun. Les lois politiques ou civiles constituant sur le registre social les pendants, en termes de droits et de devoirs réciproques, des préceptes, des règles et des devoirs de la morale, l'objet de la politique revient, positivement, à les faire observer, négativement, à empêcher les citoyens d'enfreindre les règles de l'équité en empiétant sur leurs droits réciproques ou en se dérobant aux devoirs de la morale naturelle. Aussi le gouvernement le meilleur sera, pour d'Holbach, le gouvernement le plus rationnel, celui 
capable d'aider l'être humain en tant que citoyen à se coordonner avec les causes qui agissent sur lui.

Cet eudémonisme éthico-politique délimite le cadre général $d^{\prime}$ un nouveau modèle de société: les fonctions imparties au gouvernement politique étant, comme le précise d'Holbach, de "maintenir, protéger et guider au bonheur une société $e^{8}$ ". Mais les rapports sociaux orientés par le bonheur, la mission confiée par la société à ceux qui la gouverneront pour la perpétuer et préserver son orientation, ne peuvent se réaliser sans que l'on ait spécifié, d'une part, au niveau législatif, quels sont les droits au principe de ces rapports sociaux tels qu'ils en garantissent l'exercice sous l'horizon du bonheur, et, d'autre part, sans que l'on ait déterminé, au niveau du pouvoir exécutif, la forme concrète de gouvernement, la source de l'autorité de même que les rapports réciproques entre souverains et sujets, bref tout ce qui, actualisant la nature et les objectifs $\mathrm{du}$ pouvoir, constitue les conditions objectives du bonheur dans le pacte social. Ce sera sous l'égide de la relation qui unit les trois concepts de vertu, d'utilité et de bonheur, et qui revient de façon constante chez d'Holbach pour articuler sa pensée morale et politique, qu'il faudra comprendre les interactions entre la spécification des principes mis à l'origine du modèle et les formes institutionnelles qui organisent son fonctionnement concret.

Ainsi les lois civiles ont pour objet de garantir ces droits fondamentaux que sont pour d'Holbach la liberté, la propriété et la sûreté. . Elles renvoient, d'une part, aux lois naturelles, celles qui découlent immédiatement de la nature de l'homme indépendamment de toute association, et, d'autre part elles sont définies comme exprimant les volontés de la société ${ }^{10}$. La justification du lien établi entre lois naturelles et civiles se fait alors sur la base des besoins qui sont exigés par la nature même de l'homme, besoins qui certes varient en fonction du milieu mais qui ont tous vocation de le rendre plus heureux puisque la recherche pour satisfaire les besoins est dictée par la tendance chez chacun à la conservation et au bien-être de son individu ou du corps dont il est membre.

La tendance au bonheur qui n'est donc à toutes fins prendre que la tendance chez l'homme à persévérer dans son être, et, par suite, à trouver les moyens les plus adéquats pour réussir à satisfaire ainsi ses besoins vitaux, constitue la force naturelle, la force la plus puissante qui détermine toutes les facettes de 
la vie en société. Cependant l'homme n'en est pas le jouet aveugle; si cette force est bien l'énergie même du vivant, ce vivant a aussi comme propriétés caractéristiques de raisonner et de juger. Aussi allons-nous retrouver dans les moyens que prend l'homme pour satisfaire les besoins de son être et atteindre ainsi la fin suprême du bonheur, les marques diversifiées de sa maitrise et de son appropriation des forces naturelles constitutives de son organisation. La société apparaît ainsi comme la réalisation la plus éclatante tant de la nature que de sa nature. C'est le passage même de l'état d'individu à l'état d'associé pour optimiser l'état naturel de satisfaction auquel on vise, qui nécessite de statuer sur les droits et les obligations réciproques entre la société et ses membres et aussi bien de convenir de la forme la plus appropriée de gouvernement comme des relations entre le souverain et les sujets.

L'autorité de la Société sur ses membres tire sa légitimité des avantages qu'elle leur procure et qui doivent être supérieurs aux bénéfices que l'homme aurait retiré s'il avait continué de vivre en dehors d'une association. On voit ici comment la notion d'intérêt et d'utilité se croise à celle du bonheur ou du bien-être considéré comme fin de la vie en société. L'intérêt au sens naturaliste où l'entend $\mathrm{d}^{\prime} \mathrm{Holbach}$ se confondant avec les actions prises pour accomplir son bien-être, le concept est défini de manière constante comme ce qui oriente ce bienêtre même que l'homme recherche: «en général l'intérêt d'un homme est ce qu'il juge nécessaire à sa propre félicité ${ }^{11}$ ». On expliquera de la même façon la remise des droits de la société à un ou plusieurs citoyens: c'est pour pallier aux difficultés inévitables qui surgissent du choc des volontés, des passions et des intérêts opposés et qui freineraient le développement du bien-être général que les citoyens forment un gouvernement et lui remettant l'autorité, le rendent interprète de leurs volontés pour veiller au bonheur de tous: «le gouvernement, pose d'Holbach ${ }^{12}$, est la somme des forces de la société déposées entre les mains de ceux qu'elle a jugé les plus propres à la conduire au bonheur".

Les passions pour être fructueuses, c'est-à-dire pour qu'elles aboutissent au résultat visé, maintien ou maximisation du bienêtre, doivent donc être orientées par l'intérêt ou plus précisément par la reconnaissance par le citoyen de ce qui constitue son intérêt; elles seront alors réputées utiles dans la mesure où elles auront permis la satisfaction du désir et l'atteinte du bonheur. La tendance au bonheur agit tout autant sur les relations réciproques qui lient les activités diverses des mem- 
bres d'une société en tant que citoyens. Au niveau d'abord des obligations mutuelles qu'ont contractées les uns envers les autres les membres du pacte social: si la société a des droits, elle a aussi des devoirs qui sont de faire respecter à chacun les droits de tous et de se montrer fidèle à ses engagements; et réciproquement le citoyen est obligé, en vue du bien-être et de la conservation de tous de se soumettre à la société, de la servir de ses talents et de son travail, de subordonner ses intérêts personnels à ceux de la société et de défendre son intégrité. Le bonheur que l'on retirera fonde la notion de société juste et la possibilité, du côté des citoyens, de révoquer le pacte si la société ne parvient pas à lui procurer le bonheur (liberté, sûreté, biens divers, travail...) qu'elle s'est engagée à lui procurer, tandis que, du côté de la société, les citoyens qui ont été désignés comme les interprètes des volontés de tous peuvent contraindre les citoyens récalcitrants à respecter ces volontés et ces lois. Suivant la même logique, le souverain est seulement le gardien et le dépositaire du contrat social, son exécuteur, et d'Holbach lui refuse le droit "de l'anéantir ou de le violer». Dans cette perspective le souverain n'est pas le maître, mais bien, comme l'affirme nettement d'Holbach, "le ministre de la société, chargé de remplir ses engagements envers les citoyens, et muni du pouvoir nécessaire pour obliger ceux-ci à remplir les leurs ${ }^{13} \%$. C'est donc un intérêt éclairé par la raison qui engage le citoyen à se soumettre aux lois justes d'une société lorsque celle-ci s'occupe du bien-être de ses membres: en obéissant le citoyen travaille à son propre bonheur. De la même façon ce principe sert à déterminer les limites de l'obéissance au Souverain et la légitimité de la rébellion. L'obéissance ne devant jamais être aveugle, dès que l'on s'aperçoit que le Souverain substitue sa volonté à la volonté publique, on est autorisé à lui résister et à le démettre. Le discours IV de la Politique naturelle qui traite des sujets, et entre autres de l'obéissance, déclare, reprenant ici l'inspiration lockéenne que: «Le citoyen n'obéit qu'à ce que l'Autorité a droit de lui commander, et jamais l'autorité n'a droit de rien commander de contraire à la nature, à la justice et au bien-être d'un tout auquel elle est subordonnée ${ }^{14}$ ».

L'action vertueuse, tout comme les objets de l'intérêt ou ceux du bonheur, se définit au moyen de la boussole de l'utilité qui indique toujours la direction du bien-être. De manière générale on dira, en liant explicitement vertu, bonheur et utilité que «la vertu est une disposition habituelle à faire ce qui 
contribue au bonheur des êtres de notre espèce, et à s'abstenir de ce qui peut leur nuire "; et un peu plus loin, d'Holbach ajoute: "nous n'appellerons vertu que ce que l'expérience, la réflexion, la raison nous montreront en tous temps, en tous lieux conformes à l'utilité générale et réelle des habitants de la terre ${ }^{15} »$.

Ainsi le but du bonheur qui est commun à ce qu'est l'homme individuel par nature et à ce qu'il doit être en société réunitil la politique à la morale et réciproquement la morale à la politique; la règle de l'utilité servant à fixer les droits et les devoirs qui soutiennent le jeu des rapports sociaux, au double registre individuel et collectif, sous l'horizon du bonheur. En effet, ce sont ces droits et ces devoirs qui régularisent le sens de l'action vertueuse, individuelle ou civique, à la jonction du bonheur et de l'utile. Vertu-utilité-bonheur, tels sont en somme les trois opérateurs qui permettent le fonctionnement harmonieux du modèle éthico-politique de $\mathrm{d}^{\prime} \mathrm{Holbach}$ aux points nodaux de ses articulations. En même temps qu'ils font l'enchaînement des séquences de la vie en société, ils réalisent au niveau des hommes-citoyens cette moralisation de la politique articulée réciproquement à une politicisation de la morale qui caractérise l'art de gouverner selon d'Holbach. Et, dans un sens méthodologique, ces concepts forment comme autant de repères qui nous aideront à dégager les axes de démarcation entre les formes existantes d'organisation sociale et celle que préconise d'Holbach; chemin faisant, nous poserons en regard de chacune de ces lignes de rupture, les articles de la Déclaration autour desquels se redéfinit l'institution politique nouvelle et se concrétise la réappropriation révolutionnaire des revendications philosophiques.

Gouverner, c'est maintenir, protéger et guider au bonheur une société; ce qui ne peut avoir lieu sans faire concourir tous ses membres à l'utilité générale, et sans réprimer les passions capables de nuire à la félicité de tous. D'où il suit que le gouvernement n'a pour objet que d'exciter les hommes réunis en Société à exercer entre eux les vertus sociales, ou à mettre en pratique les règles dont la morale leur fait sentir la nécessité pour leur propre intérêt ${ }^{16}$. 


\section{Le " credo du nouvel âge ${ }^{17}$ "}

Godechot $^{18}$ regroupait sous deux classes les articles de la Déclaration: les droits de l'Homme, les droits de la Nation. Le premier groupe, auquel ressortissent les articles II, IV, VII, VIII, IX, X, XI, XVII, met en relief la liberté, l'égalité, la propriété, la sûreté et la résistance à l'oppression. Le deuxième groupe est relatif, avec les articles III, VI, XII, XIII, XIV, XV, XVI, à la souveraineté nationale, au droit de faire la loi, à la justice et à la procédure criminelle, à l'organisation de la force publique, au vote de l'impôt, à la représentation et au pouvoir du citoyen de demander des comptes aux pouvoirs publics.

Comparons, d'abord, au long de la première ligne de rupture entre le modèle que le Philosophe met en place et le régime du pouvoir absolu qu'il dénonce, le "recueil " par les articles relatifs aux "droits de la nation " des considérations de d'Holbach concernant le gouvernement et sa forme. Il s'agit des affirmations essentielles, proprement politiques, de la Déclaration: le principe de la souveraineté réside dans la nation (article III), la loi est l'expression de la volonté générale (article VI), et enfin la séparation des trois pouvoirs (article XVI) qui place le pouvoir législatif dans les mains des citoyens ou de leurs représentants (article VI).

La forme de gouvernement que d'Holbach considère la plus appropriée se rencontre à l'avance avec les auteurs de la Déclaration, ce qui n'est guère étonnant puisque leur inspiration commune provient de la lecture de Montesquieu. Il s'agit de la monarchie limitée ou plus précisément d'un « gouvernement mixte " comme d'Holbach l'appelle: le pouvoir est partagé entre le souverain (qui n'est pas nécessairement un monarque) et les corps intermédiaires (diètes, états ou parlements) formés de représentants choisis parmi les citoyens. D'Holbach regarde cette forme de gouvernement "comme le chef-d'oeuvre de l'esprit humain " car sous l'autorité de "lois invariables qui commandent également à tous les membres de la société", c'est le gouvernement le plus soucieux d'éclairer les hommes sur leurs véritables intérêts, de les conduire au bonheur en protégeant leurs personnes, leurs propriétés, leurs libertés, et de les obliger à la vertu ${ }^{19}$. Sa définition du Souverain en découle qui présente celui-ci, quel que soit la forme de gouvernement à laquelle les citoyens auraient consenti, comme: "[un] citoyen à qui les nations ont conféré le droit de les gouverner pour leur propre félicité20 ${ }^{20}$. Il s'ensuit, d'une part, que l'autorité 
du souverain tire sa légitimité de ce consentement même, d'autre part que tout pouvoir est limité par les buts fondamentaux que la société se propose: se conserver, se perpétuer, progresser dans la félicité21. Quant aux représentants, ils ont pour fonctions de prévenir les abus de l'autorité souveraine en veillant à défendre les intérêts de leurs constituants et en faisant respecter les volontés générales des sujets ${ }^{22}$.

D'Holbach s'oppose donc à une monarchie absolue mais ne remet pas en cause le principe même de la monarchie principalement en raison du critère utilitariste qui ne permet pas de définir a priori le système idéal et qui renvoie à l'expérience la tâche de décider. Enfin il appartient aux gouvernés, comme on l'a vu, de décider si leur consentement est ou non un marché de dupes.

La deuxième ligne de rupture se profile dans la façon d'envisager la nature, l'objet et la fonction des lois civiles ou positives à l'intérieur du pacte social. À l'instar des autres Philosophes, toutes tendances confondues, de Montesquieu à Rousseau, comme le feront les articles IV à XI de la Déclaration relatifs à la nature et à l'extension des lois, d'Holbach proclame la transcendance des lois à laquelle tous les citoyens sans exception doivent être soumis puisque les lois sont l'expression de la volonté générale de la société et que, sans elles, nous ne pouvons nous rendre heureux. Au-dessus de nous, ces lois sont pourtant notre ouvrage puisqu'elles découlent des réflexions que nous nous faisons sur notre propre nature et qu'elles sont fondées sur les besoins et les propriétés de notre nature. Lois naturelles ou lois civiles sont les mêmes par rapport au principe et au but; les différences enregistrées dans le langage ne traduisent que la diversité d'application des lois ou encore les moyens divers de parvenir à la même fin. Les lois, prenant pour base l'utilité générale ont pour fonctions de rendre heureux le plus grand nombre d'individus. Suivant les besoins de l'État, et ensemble de notre nature, les lois permettront ce qui est juste et défendront ce qui est injuste; sera donc légitime et licite tout ce qui est conforme à la nature - et qui constituera ainsi les droits et les devoirs respectifs des gouvernants et des sujets -, injuste et illicite ce qui contredit la nature ${ }^{23}$. La législation fondée donc sur la nature, la justice, l'utilité et l'intérêt véritable de la société fera régner partout la liberté, encouragera le travail, réglera les moeurs, incitera le citoyen à la vertu, le protégera lui et ses biens et récompensera les actions louables ainsi que les talents nécessaires à la Société ${ }^{24}$. 
Mais la réflexion sur la loi s'entrelace inévitablement à celle sur la liberté et à la place essentielle que cette notion doit tenir dans une société reconstruite sur des bases politiques nouvelles. Dans la Déclaration de 1789, aussi bien que pour d'Holbach, la liberté a prééminence parmi «les droits de la personne " par son rapport privilégié à la loi à laquelle elle reste cependant entièrement soumise. D'Holbach en fait la condition d'accès à tous les autres biens, la propriété, la sûretée ${ }^{25}$, la vertu car il la définit comme la plus forte des passions de l'homme. Il se montre partisan farouche de la liberté de penser, de parler et d'écrire qui doit être inconditionnelle; ces libertés sont le soutien de tout bon gouvernement et constituent d'ailleurs l'intérêt bien compris de l'autorité politique ${ }^{26}$. L'amour de la liberté comme toutes les autres passions est fondé sur le désir de conservation mais se lie plus étroitement encore au désir de bonheur puisque l'homme épris de liberté emploie toutes ses facultés à faire sauter les obstacles qui empêcheraient son existence $d$ 'être heureuse. La liberté absolue est cependant une chimère: alors même que l'homme vivrait seul, sa liberté serait limitée par la raison et par l'intérêt de sa propre conservation. Dans l'état de société, la liberté qui se définit comme «la faculté de faire pour son bonheur tout ce que permet la nature de l'homme en société27 " est donc avantageuse à tous les citoyens. La liberté du citoyen trouve ses limites soit par l'équité naturelle qui lui défend de nuire aux autres, soit par des lois positives qui l'obligent à observer ses devoirs envers ses associés. "Le bien de la société totale doit donc être la mesure de la liberté de ses membres ${ }^{28}$ » affirme d'Holbach.

La liberté ne signifie pas pour autant l'égalité absolue entre les citoyens: l'utilité sociale étant inégalement répartie, il y aurait là entorse au critère d'utilité29; $\mathrm{D}^{\prime}$ Holbach recommande plutôt l'établissement de lois qui remédieraient à l'inégalité naturelle des hommes en protégeant également grands et petits, et qui réduiraient la disparité des niveaux de vie entre riches et pauvres. Selon un développement similaire, la Déclaration de 1789, même si l'article I mentionne explicitement l'égalité au même niveau que la liberté ( Les hommes naissent [...] libres et égaux en droits »), s'attache surtout à situer l'égalité en fonction de la loi (la loi est la même pour tous, affirme l'article VI), à éliminer disparités et privilèges devant l'impôt (article XIII), l'appareil judiciaire ou l'accès aux places et aux emplois publics (article VI). Ne reconnaissant d'autres distinctions que celles des capacités ou des talents, le critère de l'utilité 
commune (article I) demeure le critère suprême dans le nouvel ordre social et politique.

«Sans liberté, sans propriété, sans sûreté, une nation ne peut jouir longtemps d'une puissance véritable $e^{30}$ ", conclut d'Holbach. De même l'article XVII clôt avec le droit de propriétée ${ }^{31}$, présenté comme "inviolable et sacré ", la triade des droits imprescriptibles de l'homme du nouveau régime (article II), liberté, égalité des hommes et conservation de soi-même constituant la base de l'idée de propriété, et tous principes nécessaires au bonheur social.

La contribution idéologique de $\mathrm{d}$ 'Holbach pourrait se résumer par la formule éclatante sur laquelle s'ouvre le discours IV de la Politique naturelle: "La Loi commande à des sujets; le despotisme commande à des esclaves; la tyrannie commande à des ennemis ». La redéfinition du citoyen ${ }^{32}$ qu'opère le libéralisme politique de d'Holbach, les fondements philosophiques qu'il lui foumit aura eu, en effet, partie liée avec le renouvellement des rapports que l'homme comme être sensible et raisonnable, mû par le désir de bonheur et épris de liberté, devait engager avec les forces arbitraires pour leur substituer l'autorité de la raison, le pouvoir de la vérité3 ${ }^{33}$, la suprématie de la loi, la souveraineté du peuple. Et la Déclaration intégrant au discours constitutionnel ces valeurs auxquelles aspire le nouveau citoyen aussi bien que les principes qui guident son action, s'apprête à transformer en pratiques sociales les droits civiques et politiques qu'elle proclame. Les promesses de la Philosophie semblent ainsi s'accomplir et la félicité de l'homme de la raison future garantie par la loi: liberté, égalité civique, propriété et sûreté.

Département de philosophie

Université du Québec à Montréal

\section{NOTES}

1. Ces formules sont du Discours préliminaire, un texte rarissime de 16 pages in- $8^{\circ}$, numérotées entre crochets, sans doute de la main de Naigeon, et qui est annexé à un second tirage hollandais du Système de la nature, publié en 1770 , six mois après le premier tirage du Système. Ce Discours préliminaire, a été republié de nos jours par les soins érudits de M. Jeroom VERCRUYSSE in Bicentenaire du Système de la nature. Textes holbachiens peu connus, Paris, Lettres modernes, 1970. Les propositions que nous citons se trouvent à la p. 47 de cette réédition accompagnée de notes bibliographiques. 
2. Discours préliminaire, p. 42.

3. Discours préliminaire, p. 45.

4. Système social, II, I, p. 4. Nous utilisons l'édition originale de 1773, Londres, sans nom d'auteur.

5. Système social, II, 1, p. 19. D’Holbach ajoute, en étendant le domaine des lois de la morale aux rapports entre nations, et après avoir distingué entre les objets de la politique intérieure et les objets de la politique extérieure, qu' « en un mot, la politique est la morale des nations ", ibidem, p. 20.

6. Voir notamment Système social, I, XII; II, I ainsi que Politique Naturelle, Discours I, § VI, §XXXVI. Nous utilisons l'édition originale, 1773, Londres.

7. Système social, Introduction, pp. VII-VIII. La citation complète se lit comme suit:

La morale et la politique sont évidemment liées; elles ne peuvent sans danger se séparer d'intérêts, ni cesser de se donner la main. La morale n'a point de force, si la politique ne l'appuie; la politique est chancellante et s'égare, si elle n'est soutenue et aidée par la vertu. L'objet de la morale est de faire connaitre aux hommes que leur plus grand intérêt exigent qu'ils pratiquent la vertu; le but du gouvernement doit être de la leur faire pratiquer. La morale ne peut qu'inviter les hommes à faire le bien; le gouvernement peut, ou les y contraindre par les lois, ou les y solliciter par ses récompenses et ses bienfaits. La morale ne sera pour les nations qu'une science spéculative, et ses leçons demeureront impraticables, tant que les arbitres de leurs destinées ne sentiront pas que sans vertu nulle puissance sur la terre ne peut être sûre et fortunée, et ne feront pas sentir aux citoyens que nul homme en société ne peut être heureux sans la vertu.

Permettons-nous de voir dans cette moralisation de la politique opérée par d'Holbach, au nom du bonheur et de la vertu, et qui lui permet d'articuler enseignement spéculatif et enseignement pratique, une annonce de la répétition par Robespierre et la politique morale des Jacobins, de cette liaison entre action morale et action politique; elle suit les mêmes mécanismes et la même modélisation pour justifier-rationaliser une mesure politique précise, la Terreur, au nom de la vertu et du bonheur social.

8. Système social, II, I, p. 20.

9. Ibidem, pp. 6-8; voir aussi Politique naturelle, Discours I, $\S$ VII, $\S$ VIII, $\S \times V, \S X V I, \S X V I I$.

10. Système social, II, I, pp. 6-7. 
11. Système social, I, VI, p. 64. Voir aussi, par exemple, une définition de l'intérêt comme " le désir excité par l'objet dans lequel chaque homme fait consister son bien-être ". Ibidem, p. 60 .

12. Système social, II, I, p. 6

13. Ibidem.

14. Politique naturelle, Discours IV, § III.

15. Système social, I, VIII, p. 76, p. 82.

16. Système social, II, I, p. 20.

17. La formule est de Michelet.

18. Jacques GODECHOT, Les Institutions de la France sous la Révolution et l'Empire, P.U.F., Paris, 2e éd. augmentée, 1968.

19. Voir Politique naturelle, II, $\S \mathrm{XIX}$.

20. Politique naturelle, III, $\S \mathrm{I}$.

21. Ibidem. On pourrait comparer aussi le préambule de la Déclaration qui souligne la fonction primordiale d'une Constitution: assurer le bonheur de tous.

22. Voir Politique naturelle, IV, § XIV. Cf. aussi l'article "représentants" dans l'Encyclopédie qui est de d'Holbach et où il résume vigoureusement ses propres positions sur la question en mème temps qu'il synthétise les points saillants de la pensée politique des Philosophes cristallisée autour de ce concept-clé de la lutte idéologique contre le pouvoir absolu.

23. L'article IV énonce: “ La liberté consiste à faire tout ce qui ne nuit pas à autrui: ainsi l'exercice des droits naturels de chaque homme n'a de bornes que celles qui assurent aux autres membres de la société la jouissance de ces mêmes droits. Ces bornes ne peuvent être déterminées que par la loi », et se précise par l'article V: "La Ioi n'a le droit de défendre que les actions nuisibles à la société. Tout ce qui n'est pas défendu par la loi ne peut être empêché, et nul ne peut être contraint à faire ce qu'elle n'ordonne pas ". Ce rapport de la liberté à la loi comme référent suprême est constant chez les Philosophes depuis Montesquieu (Esprit des Lois, Livre XI, ch. 3); $d^{\prime}$ Holbach le fonde sur sa trilogie conceptuelle éthico-politique, utilité-bonheur-vertu mais il faudra attendre Kant pour la radicalisation par le sujet de l'action et la loi qu'il intériorise, du sujet du droit.

24. Voir Politique naturelle, I; IX, $\S$ XXV. Comparer aussi le contenu de l'article VI de la Déclaration.

25. Voir Politique naturelle, VI, $\S \mathrm{XI}$.

26. Système social, II, ch. V; Politique naturelle, VI, § IX-XXVI. Voir les articles $X$ et $X I$ de la Déclaration qui garantissent l'exercice de ces mêmes libertés et les placent sous l'autorité de la loi.

27. Politique naturelle, VI, $\$$ II; voir aussi Système social, II, ch. III pour tout ce qui concerne la trilogie holbachienne, liberté, propriété, 
sûreté. On sait aussi le souci central chez les auteurs de la Déclaration de faire dépendre de la loi, liberté-bien public-bonheur.

28. Politique naturelle, VI, § II. Voir aussi ici l'article IV de la Déclaration.

29. Système social, II, III, p. 41,

30. Politique naturelle, VI, $\&$ XXIII.

31. Chez d'Holbach, comme dans la doctrine de Locke, c'est la propriété qui fait le citoyen. D'Holbach écrit, défendant le mode d'appropriation bourgeois fondé sur le travail: « [...] les lois de la Nature donnent à chaque homme un droit que l'on appelle propriété, qui n'est que la faculté de jouir exclusivement des choses que le talent, le travail et l'industrie procurent ", in Politique Naturelle, I, $\$$ XXV.

32. Ainsi que l'exhortait d'Holbach dès l'Abrégé du code de la nature (II, 14, p. 234):

Sois citoyen, parce que ta patrie est nécessaire à ta sûreté, à tes plaisirs, à ton bien-être. Sois fidèle et soumis à l'autorité légitime parce qu'elle est nécessaire au maintien de la société qui t'est nécessaire à toi-même. Obéis aux lois parce qu'elles sont l'expression de la volonté publique à laquelle ta volonté particulière doit être subordonnée. Défends ton pays parce que c'est lui qui te rend heureux et qui renferme tes biens ainsi que tous les êtres les plus chers à ton coeur. Ne souffre point que cette mère commune de toi et de tes concitoyens tombe dans les fers de la tyrannie, parce que pour lors elle ne serait plus qu'une prison pour toi. Si ton injuste patrie te refuse le bonheur, si soumise à un pouvoir injuste, elle souffre qu' on t'opprime, éloigne toi d'elle en silence, ne la trouble jamais.

33. Le Philosophe milite pour le changement de régime politique en prônant le progrès de la raison et la substitution du savoir à la croyance. C'est à cette lumière qu'il faut évaluer la définition de la vérité telle que la formule d'Holbach: plutôt que d'en donner une définition universelle et de lui attribuer un sens a priori, il essaie de mettre en place un ordre de vérités. La vérité sera toujours accessible pour l'homme qui la cherche à la fois dans ses causes et ses effets, elle est le fruit dynamique des lois de la nature, et c'est à l'homme encore à qui il appartiendra de mettre en place les conditions de cette adéquation nnur retrouver le juste mouvement de la vérité, reconnaître le parcours où elle s'exerce et en déterminer les limites. D’Holbach écrit dans le Système social, I, I, 11: «La vérité en Physique est la connaissance des effets que les causes naturelles doivent produire sur nos sens. La vérité en Morale est la connaissance des effets que les actions des hommes doivent produire sur les hommes. La vérité en politique est la connaissance des effets que le Gouvernement produit sur la Société, c'est-à-dire la manière dont il influe sur la félicité publique et particulière des citoyens ". 